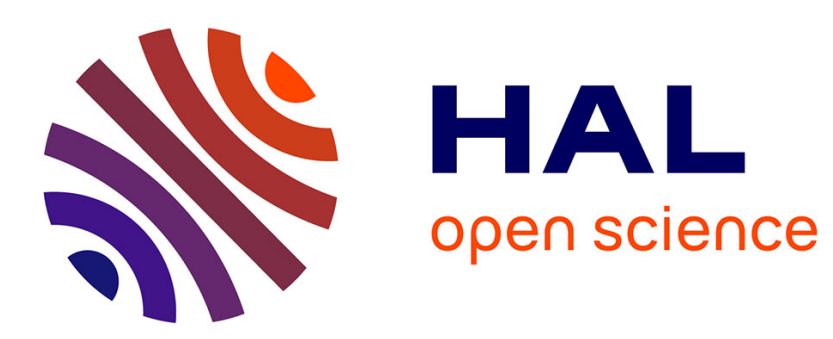

\title{
Matching Theory for Future Wireless Networks: Fundamentals and Applications
}

Yunan Gu, Walid Saad, Mehdi Bennis, Merouane Debbah, Zhu Han

\section{To cite this version:}

Yunan Gu, Walid Saad, Mehdi Bennis, Merouane Debbah, Zhu Han. Matching Theory for Future Wireless Networks: Fundamentals and Applications. IEEE Communications Magazine, 2015, 53 (5), pp.52 - 59. 10.1109/mcom.2015.7105641 . hal-01242451

\section{HAL Id: hal-01242451 \\ https://hal.science/hal-01242451}

Submitted on 12 Dec 2015

HAL is a multi-disciplinary open access archive for the deposit and dissemination of scientific research documents, whether they are published or not. The documents may come from teaching and research institutions in France or abroad, or from public or private research centers.
L'archive ouverte pluridisciplinaire HAL, est destinée au dépôt et à la diffusion de documents scientifiques de niveau recherche, publiés ou non, émanant des établissements d'enseignement et de recherche français ou étrangers, des laboratoires publics ou privés. 


\title{
Matching Theory for Future Wireless Networks: Fundamentals and Applications
}

\author{
Yunan Gu, Walid Saad, Mehdi Bennis, Merouane Debbah, and Zhu Han
}

Yunan Gu and Zhu Han are with the University of Houston.

Walid Saad is with Virginia Tech.

Mehdi Bennis is with the University of Oulu.

Merouane Debbah is with Huawei France $R \& D$.

The work of Yunan Gu and Zhu Han was supported by the U.S.

National Science Foundation under Grants CMMI-1434789, ECCS1405121, CNS-1443917, CNS-1265268, CNS0953377, and NSFC 61428101. The work of Walid Saad was supported by the U.S. National Science Foundation under Grants CNS1460316, CNS-1460333, CNS-1443917, ECCS1405121, CNS-1265268, and CNS-0953377.

\begin{abstract}
The emergence of novel wireless networking paradigms such as small cell and cognitive radio networks has forever transformed the way in which wireless systems are operated. In particular, the need for self-organizing solutions to manage the scarce spectral resources has become a prevalent theme in many emerging wireless systems. In this article, the first comprehensive tutorial on the use of matching theory, a Nobel Prize winning framework, for resource management in wireless networks is developed. To cater for the unique features of emerging wireless networks, a novel, wireless-oriented classification of matching theory is proposed. Then the key solution concepts and algorithmic implementations of this framework are exposed. The developed concepts are applied in three important wireless networking areas in order to demonstrate the usefulness of this analytical tool. Results show how matching theory can effectively improve the performance of resource allocation in all three applications discussed.
\end{abstract}

\section{INTRODUCTION}

Smartphones, tablets, and other handheld devices are exponentially increasing the traffic load in current wireless networks. To meet this increasing demand, several new paradigms have emerged:

- Cognitive radio (CR) networks, in which cognitive devices can adaptively opportunistically access the wireless spectrum, thus improving spectral utilization

- Small cell networks that boost wireless capacity and coverage via a viral deployment of low-cost small cell base stations

- Large-scale device-to-device communications that can occur over both cellular and unlicensed bands

This is gradually leading to a future multi-tiered heterogeneous wireless architecture, as seen in Fig. 1.
Effectively managing resource allocation in such a complex environment warrants a fundamental shift from traditional centralized mechanisms toward self-organizing and self-optimizing approaches. The need for this shift is motivated by practical factors such as the increasing density of wireless networks and the need for communications with low latency. Even recent emerging centralized paradigms such as cloud-based radio access networking (RAN) will still require some form of self-organization due to country-specific backhaul constraints. In consequence, there is a need for self-organizing systems in which small cell base stations and even devices can have some intelligence to rapidly make resource management decisions.

Indeed, there has been a recent surge in the literature that proposes new mathematical tools for optimizing resource allocation in many emerging wireless systems. Examples include centralized optimization and game theory. Centralized optimization techniques can provide optimal solutions to resource allocation problems, and their algorithmic implementations have matured over the past few years. However, they often require global network information and centralized control, thus yielding significant overhead and complexity. This complexity can rapidly increase when dealing with combinatorial integer programming problems such as channel allocation and user association. Moreover, centralized optimization may not be able to properly handle the challenges of dense and heterogeneous wireless environments such as the one in Fig. 1.

The aforementioned limitations of optimization have led to an interesting body of literature that deals with the use of noncooperative game theory for wireless resource allocation [1]. Despite their potential, such approaches present some shortcomings. First, classical game-theoretic algorithms such as best response will require some form of knowledge of other players' actions, thus limiting their distributed implementation. Second, most game-theoretic solutions, 
such as the Nash equilibrium, investigate onesided (or unilateral) stability notions in which equilibrium deviations are evaluated unilaterally per player. Such unilateral deviations may not be practical when investigating assignment problems between distinct sets of players. Last but not least, the tractability of equilibria in gametheoretic methods requires having some structure in the objective functions, which for practical wireless metrics may not always be satisfied.

Recently, matching theory has emerged as a promising technique for wireless resource allocation, which can overcome some limitations of game theory and optimization [2-6]. Matching theory is a Nobel Prize winning framework that provides mathematically tractable solutions for the combinatorial problem of matching players in two distinct sets [7-9], depending on the individual information and preference of each player. The advantages of matching theory for wireless resource management include:

- Suitable models for characterizing interactions between heterogeneous nodes, each of which has its own type, objective, and information

- The ability to define general "preferences" that can handle heterogeneous and complex considerations related to wireless qualityof-service (QoS)

- Suitable solutions, in terms of stability and optimality, that accurately reflect different system objectives

- Efficient algorithmic implementations that are inherently self-organizing and amenable to fast implementation

However, reaping the benefits of matching theory for wireless networks requires advancing this framework to handle their intrinsic properties such as interference and delay. Despite the surge in research that applies matching theory for wireless, most existing works are restricted to very limited aspects of resource allocation. This is mainly due to the sparsity of tutorials that tackle matching theory from an engineering perspective. For instance, most references, such as [7-9], focus on matching problems in microeconomics. In addition, although [10] provides an interesting introduction to matching theory for engineering, it does not explicitly explore the challenges of future wireless systems.

In this tutorial, we aim to provide a unified treatment of matching theory oriented toward engineering applications in general, and wireless networking in particular. The goal is to gather the state-of-the-art contributions that address the major opportunities and challenges in applying matching theory to the understanding of emerging wireless networks, with emphasis on both new analytical techniques and novel application scenarios. Beyond providing a self-contained tutorial on classical matching concepts, we introduce a new classification that is oriented toward next-generation wireless systems. For each class of matching problems, we provide the basic challenges, solution concepts, and potential applications. Then we conclude by summarizing the potential of matching theory as a tool for resource management in wireless networks.

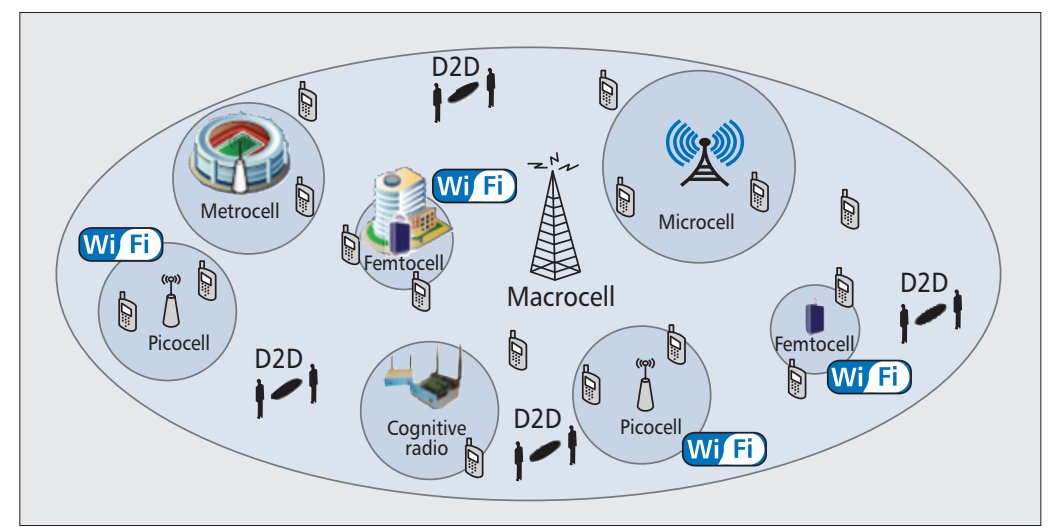

Figure 1. A future wireless network with a mixture of small cells, cognitive radio devices, and heterogeneous spectrum bands.

\section{MATCHING THEORY: FUNDAMENTALS AND Conventional Classification}

\section{Basic Matching Definitions}

The basic wireless resource management problem can be posed as a matching problem between resources and users. Depending on the scenario, the resources can be of different abstraction levels, representing base stations, time-frequency chunks, power, or others. Users can be devices, stations, or smartphone applications. Each user and resource has a quota that defines the maximum number of players with which it can be matched. The main goal of matching is to optimally match resources and users given their individual, often different, objectives and learned information. Each user (resource) builds a ranking of the resources (users) using a preference relation. The concept of a preference represents the individual view that each resource or user has of the other set, based on local information. In its basic form, a preference can simply be defined in terms of an objective utility function that quantifies the QoS achieved by a certain resource-user matching. However, a preference is more generic than a utility function in that it can incorporate additional qualitative measures extracted from the information available to users and resources.

A matching is essentially an allocation between resources and users. The basic solution concept for a matching problem is the so-called two-sided stable matching. A matching is said to be two-sided stable if and only if there is no blocking pair (BP). A BP for a stable marriage case is defined as a pair of user and resource $(u$, $r$ ) where $u$ prefers $r$ to its currently matched user $j$, and $r$ prefers $u$ to its currently matched resource $k$. Thus, $u$ will leave $i$ to be matched to $r$, and $r$ would rather be matched to user $u$ than user $k$. The implication of stability in a wireless network is further discussed later. This definition of stability can extend to all types of matching problems.

\section{Conventional Classification}

The classical classification of matching problems is based on the values of the player quotas as follows: 


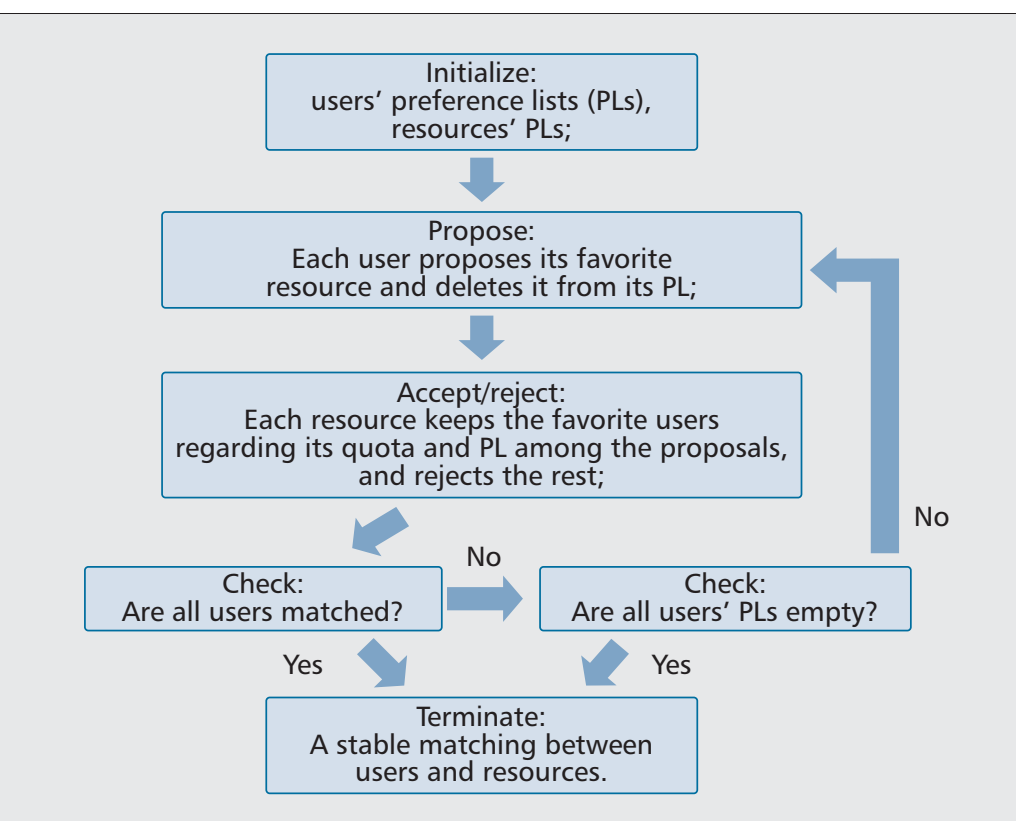

Figure 2. Deferred acceptance algorithm. many distributed implementations, which do not require the players to know each other's preferences [11]. When the preferences are strict (no indifference), the stable matching is also Pareto optimal for the proposing players [11]. Extensions that balance the roles of proposing and receiving players or handle many-to-many cases have been developed such as in $[10,12]$.

From an information exchange point of view, even though DA requires players to submit proposals to one another, it does not require a centralized controller. During the information exchange (proposals), each player is required to only collect information on the players in whom they are interested from the opposite set to perform a ranking according to its preferences. The players need not observe the actions or preferences of other players. After building preference lists, the players take actions based on the local information they have collected without requiring any synchronization in time. The convergence of DA to stable matching is guaranteed irrespective of the order of play and without any synchronization. Therefore, a DA-based approach can be implemented in a distributed manner without requiring a central information collection center. For such distributed implementations, the results on the polynomial time convergence of one-toone matching would still hold as corroborated by some recent studies $[3,13]$.

\section{MATCHING IN WIRELESS NetWorks: Fundamentals}

\section{Wireless-Oriented Classification}

To capture the various wireless resource management features, we condense the rich matching literature into three new proposed classes of problems, illustrated in Fig. 3, having the following properties:

Class I: Canonical matching: This constitutes the baseline class in which the preference of any resource (user) depends solely on the information available at this resource (user) and on the users (resources) with which it is seeking to match. This is useful to study resource management within a single cell or for allocating orthogonal spectrum resources. This is particularly applicable, for example, to CR networks, in which one must allocate orthogonal licensed channels to a number of unlicensed users.

Class II: Matching with externalities: This class allows desirable matchings to be found when the problem exhibits "externalities," which translate into interdependencies between the players' preferences. For example, in a small cell network, whenever a user is associated with a resource, the preference of other users will automatically change, since this allocated resource can create interference at other resources using the same frequency. Thus, the preferences of any player depend not only on the information available at this player, but also on the entire matching of the others. We distinguish between two types of externalities: conventional externalities and peer effects. In the former, the dependence of the preferences is between players matched to different players in the other set, such as in the interference example. In the lat- 


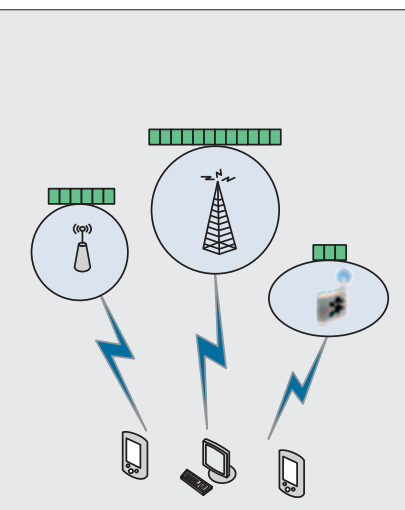

Class I - Canonical matching games:

- Example application: Allocation of orthogonal spectrum in cognitive radio networks

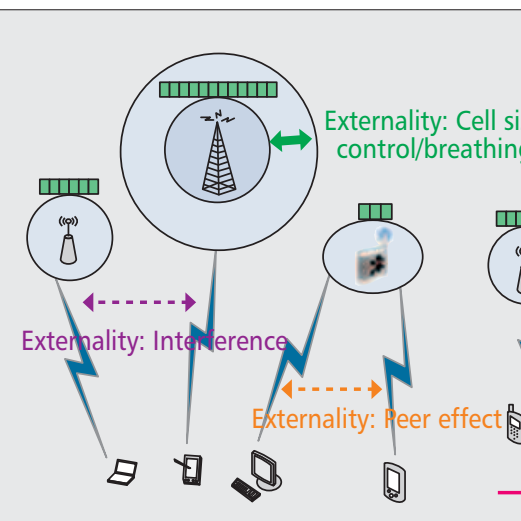

Class II - Matching with externalities:

- Example application: Proactive cell association, context-aware allocation, interference management, and load balancing

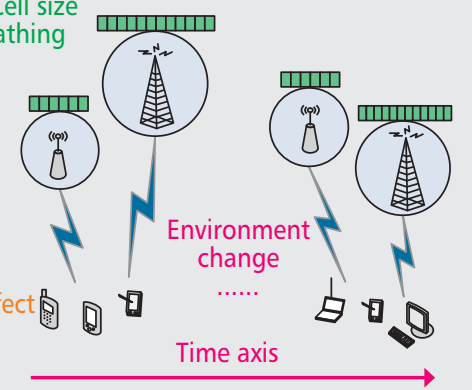

Class III - Matching with dynamics:

- Example application: Resource management with environmental variations

Figure 3. Novel wireless-oriented classification of matching theory.

ter, the preference of a user for a resource will depend on the identity and number of other users matched to the same resource. Such peer effects are abundant in a wireless environment due to factors such as delay.

Class III: Matching with dynamics: The third class, matching with dynamics, is suitable for scenarios in which one must adapt the matching processes to dynamics of the environment such as fast fading, mobility, and time-varying traffic. Here, at each time, the preferences of the players might change; thus, the time dimension must be accounted for in the matching solution. However, for a given time, the matching problem can be of either class I or class II.

Mathematically, the formulation of problems in all three classes will follow the basics of an earlier section. For class I, the preferences of one player set simply depend on the other player set. However, for class II, the preferences will now depend not only on the matched user, but also on the entire matching, due to externalities. For class III, one can introduce a time-dependent state variable in the matching. Subsequently, the preferences will now be time- and state-dependent if the problem has both dynamics and externalities. The transition between states depends on the application being studied. For example, if the state represents the activity pattern of a licensed user, the transition would follow a classic Markov model. In contrast, if the state represents a dynamically varying fast fading channel, one can use differential equations to represent the state transition.

\section{Matching Theory in WiReless: Discussions}

In wireless resource management, the matching stability notion discussed earlier implies robustness to deviations that can benefit both the resource owners and the users. In fact, an unstable matching can, for example, lead to undesirable cases in which a base station can swap its least preferred user with another since this swap is beneficial to both the resource and the user. Having such network-wide deviations ultimately leads to an unstable network operation. Remark- ably, a recent result [2] has shown that classical schemes such as proportional fair often yield unstable matchings, which further motivates the need to analyze and optimize stable matchings for self-organizing wireless systems. This concept is very useful in matching problems and is broadly applicable to all classes. ${ }^{1}$

While the existence of a stable matching is guaranteed for canonical games in the one-toone and one-to-many cases, such results do not readily map to many-to-many or to classes II and III. However, although DA and its variants were originally conceived for canonical matching, one can also use them as the nexus of new matching algorithms, tailored to the nature of wireless networks. Such algorithms can be used to establish the existence of stable matchings for classes II and II as well as to find outcomes with desirable efficiency properties.

Here, we note that there is no general existence result for stable matching with externalities. However, to handle externalities, one can utilize an iterative DA process that continuously updates the preferences based on the currently perceived matching. By exploiting the structure of externalities via wireless concepts such as interference graphs (e.g., who interferes with whom), one can analyze the convergence and stability of the resultant matching. Naturally, by building on such methods one can expand the realm of matching theory to handle externalities. Similarly, by integrating notions from stochastic games into matching, practical dynamic algorithms can be devised to find matchings that can cope with time-varying changes and are stable over time. The basic idea is to cast the matching problem as a stochastic game and then explore the rich literature on dynamic game theory [1] to solve this problem while ensuring that the solution will converge to a two-sided stable matching rather than a classical Nash equilibrium. The solution would now essentially be a dynamic and stochastic version of DA.

Although the above discussed matching solutions could provide stable matchings, they also have some limitations:
The seminal result in matching theory shows that at least one stable matching exists for general preferences in conventional one-to-one and one-to-many games. This work also introduced an efficient algorithm, known as the deferred acceptance algorithm (polynomial time for one-toone and empirically very fast for one-tomany), which can find such a matching.
1 This concept can also be connected to other stronger or weaker stability notions (e.g., setwise or Nash stability) that have various interpretations. 


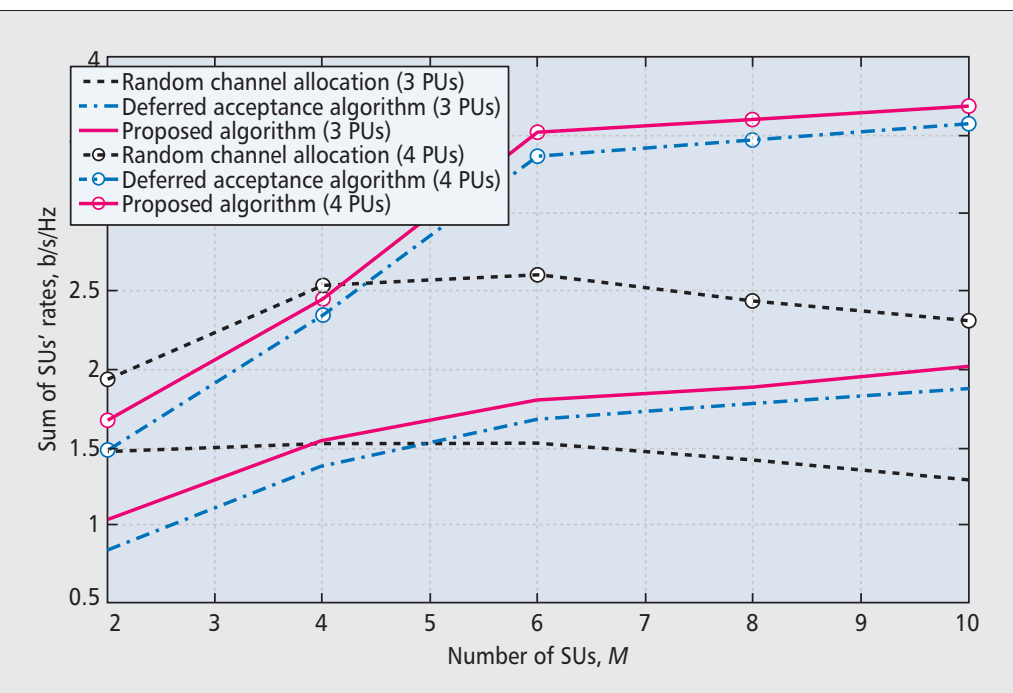

Figure 4. Example result showing how matching improves channel allocation in cognitive radio networks.

- Similar to game theory, matching problems can admit multiple stable solutions; thus, the selection of a desirable matching is a key design issue.

- The optimality of the stable solution may not be always guaranteed; however, one can utilize known techniques, such as pricing or optimized utility designs, to drive the matching solution toward an optimal and stable point,

- The exchange of proposals during DA requires additional signaling in a wireless network; however, one can exploit some structure of the problem to reduce the number of proposals as done in [3].

\section{MATCHING ThEORY IN WIRELESS NETWORKS: APPLICATIONS}

\section{Cognitive Radio Networks}

Cognitive radio networks present a primary application of matching theory due to:

- The necessity of decentralized operation

- The need for dynamic spectrum access, which requires efficient resource management solutions

- The two-sided nature of CR in which licensed primary users (PUs) own channels that must be accessed by unlicensed secondary users (SUs)

Indeed, in CR networks, centralized optimization solutions are undesirable since PUs and SUs often belong to different operators and cannot be centrally controlled. On the other hand, in some CR problems such as PU-SU association, using a noncooperative game can lead to stable solutions in which matching PUs and SUs is done without requiring the two-sided consent of both PUs and SUs.

The suitability of matching theory for CR has been corroborated by a number of recent works such as [3, 4]. In particular, [3] presented one of the first works in this area. In this work, a oneto-one matching problem is formulated between a number of SUs and a number of PUs (chan- nels). The channels are assumed to be orthogonal; hence, the game is a canonical matching game. The preferences of both users and channels are based on the same utility function, which primarily captures the rate of transmission. Under this model, it is shown that:

- A unique stable matching exists.

- A modified version of the DA algorithm can be used to find the stable allocation in a time efficient manner.

This work was extended in [4] to account for energy efficiency.

Recently, we also studied a one-to-one matching problem between SUs and PUs in which the SUs rank the PUs based on their confidence in sensing the PUs' channels. In particular, using a soft-decision Bayesian framework, we quantified the accuracy of the sensing of each channel and incorporated this metric into the SUs' preferences. Prior to matching, each SU evaluates its appreciation of the PU channel by capturing the effect of confidence in sensing as well as rate. Then the PUs actively participate in the association process based on two cases:

- When inactive, the PU prefers to grant its band to the SU with the highest sensing detection and rate to better exploit the channel.

- When active, the PU prefers to protect its band, and thus will attempt to limit or deny association.

Here, we show that the matching is canonical and adopt a modified DA algorithm that allows the PUs to handle the aforementioned property. As shown in Fig. 4, for the studied scenario, the matching-based algorithm yields significant performance gains, in terms of the SUs sum rate, when compared to classical random channel allocation schemes. Moreover, the modified DA algorithm also presents sum rate improvements over classical DA (similar gains can be seen in terms of convergence time).

Clearly, CR networks present an important avenue for matching theory. Many extensions to the existing works can be envisioned, particularly by exploring matching with externalities (under interference constraints) and dynamic matching (given time-varying PU activity).

\section{HETEROGENEOUS Small-Cell-Based Networks}

Heterogeneous (small-cell-based wireless) networks (HetNets) present an important application of matching theory due to their heterogeneity and scale. Also, there has been increased interest recently in developing contextaware or user-centric HetNets that can exploit new dimensions such as social metrics to improve resource allocation. Such context awareness further motivates the need for distributed solutions that account for the individual context available at each node - similar to how matching captures individual preferences. Given this striking analogy between matching theory and resource management in HetNets, the proposed classes can be used to address a variety of problems, which include interference management, handover management, caching, and cell association.

Here, matching is preferred over optimiza- 

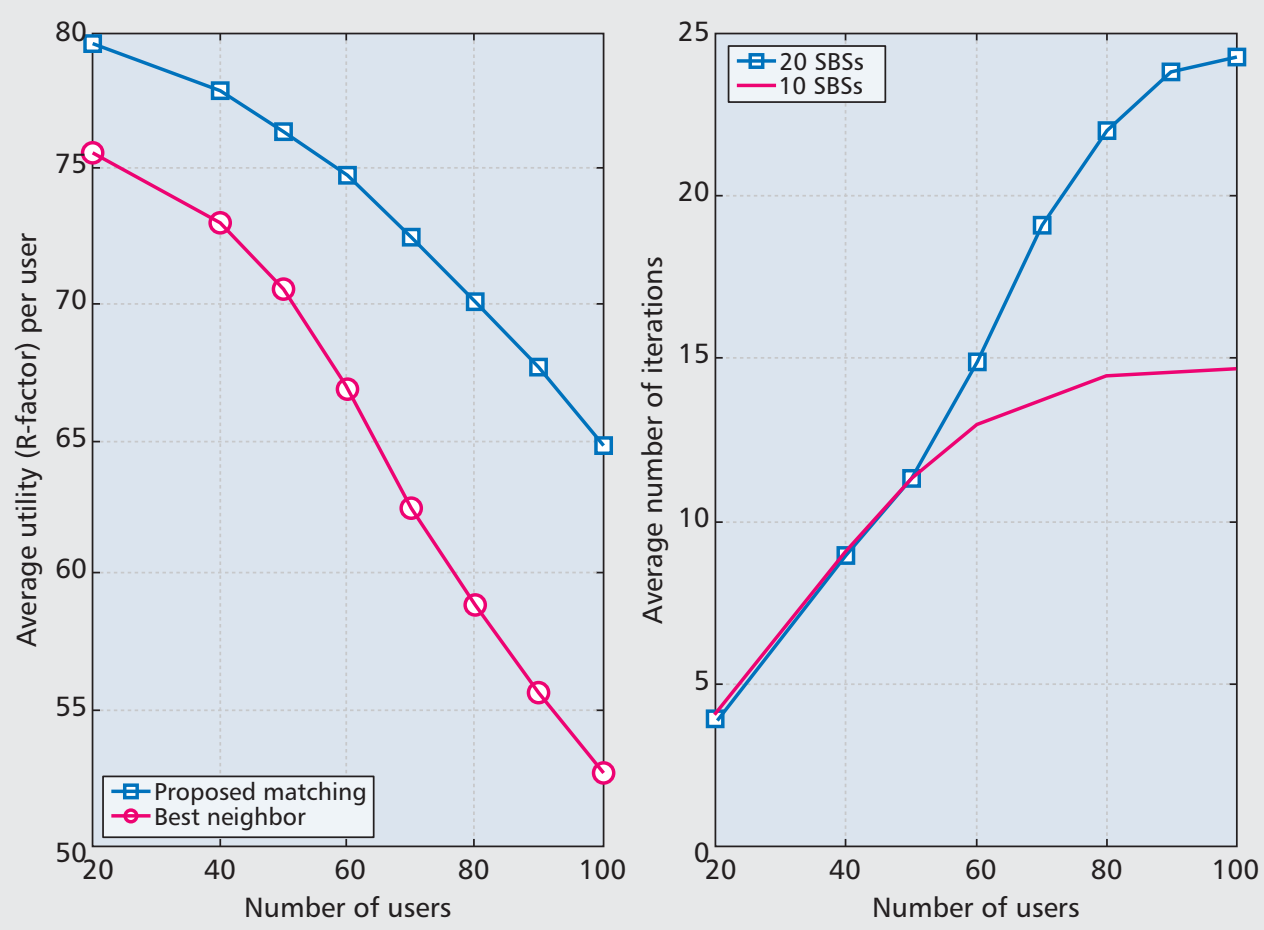

HetNets present an

important application of matching theory due to their heterogeneity and scale. Also, there has been an increased recent interest in developing context-aware or user-centric HetNets that can exploit new dimensions such as social metrics to improve resource allocation.

Figure 5. Example result showing how matching theory can be used to improve uplink cell association in HetNets.

tion due to:

- The density and scale of HetNets, which motivate self-organizing solutions

- The need to account for the context present at each small cell base station (SBS) and device instead of a single global utility function

- The centralized optimization approach, which will generally yield a combinatorial problem, particularly, in the presence of heterogeneous context, which limits its applicability here

Moreover, although a noncooperative game is also applicable here, it will have a number of limitations that include the need to observe (at least partially) all players' preferences and the fact that the solution concepts would not account for two-sided stability as previously explained.

In [14], we studied the problem of cell association in the uplink of a HetNet. The basic model here is an uplink HetNet model in which each user needs to decide to which SBS it should be assigned. The problem is formulated as a oneto-many matching model in which a user can be associated with only one SBS, and an SBS can admit a certain quota of users. The users' preferences over SBSs capture the bit error rate and delay trade-off that they can achieve, while the SBSs' preferences favor load balancing by pushing users to the smaller cells without jeopardizing QoS. Such a load balancing is essentially a form of cell biasing in which an SBS would offload some users from the macrocell and service them directly. Here, we also consider the delay at each SBS due to the increasing load and the limited capacity of the backhaul that connects the SBSs to the core network. Therefore, although orthogonal spectrum is considered, due to the delay, the matching problem is shown to have peer effects, and thus belongs to class II, matching with externalities.

Due to peer effects, applying DA and its variants will not yield a stable matching. Instead, we developed a new algorithm that starts with a distributed DA-based process using initial preferences based on the worst case delay. Then, as the nodes measure externalities, they modify their preferences and change their choices by transferring to other SBSs. Then we show that due to the presence of transfers in the model, delay-sensitive users will trade-off two-sided stability for a weaker stability that achieves better QoS. Figure 5 shows simulation results for 2 macrocells and 10 SBSs. We can see that the matching-based approach outperforms the benchmark best neighbor scheme often adopted in classical cellular systems, with up to 23 percent improvement in average user utility. Figure 5 also shows a reasonable convergence time that grows slowly with network size.

One can extend this framework of matching with peer effects or, more generally, externalities to many other areas in HetNets. For example, in [5], the framework is extended to account for interference and perform downlink cell association for a context-aware network in which preferences capture a palette of information including application type, hardware size, and physical layer metrics. In addition, as shown in in [13], one can explore canonical matching models to study the association not only at the radio level, but also at the level of operators.

In a nutshell, matching-theoretic models, in all three classes, can serve as a fundamental ana- 


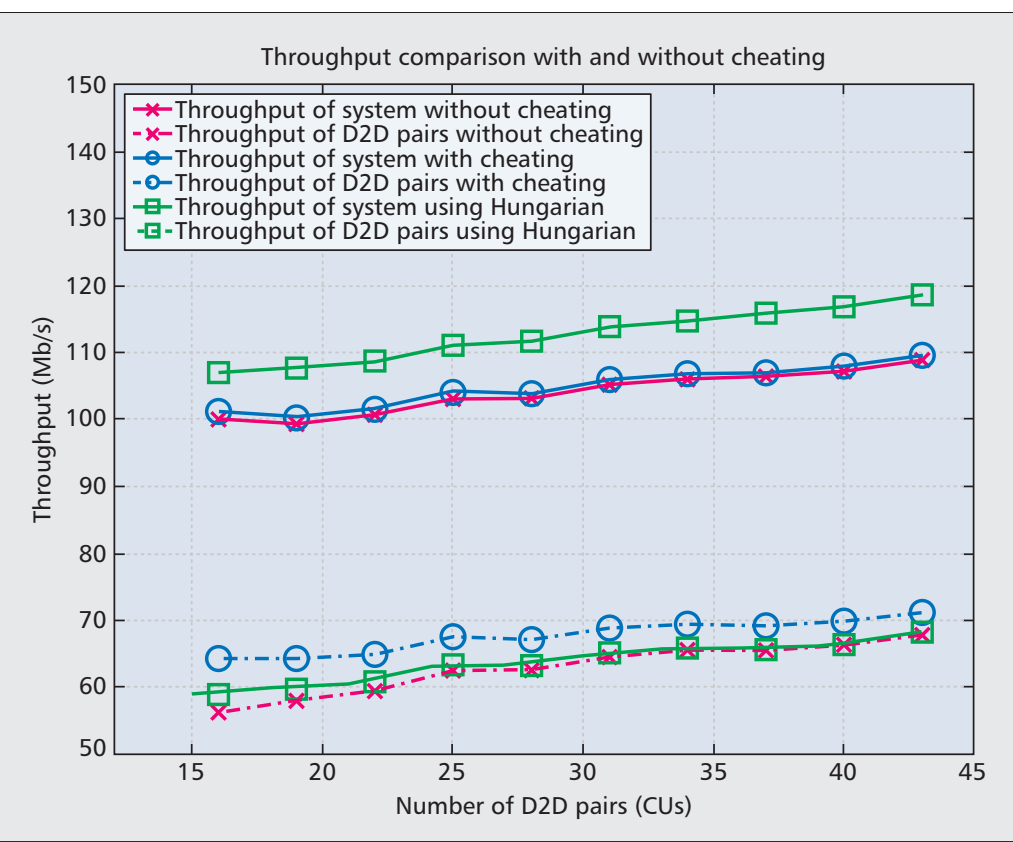

Figure 6. Example result showing how cheating further improves both DU and system utilities. share the spectrum with the CUs.

Here, we formulate a two-sided matching problem between the CUs and DUs. Each CU and DU starts by building its preference list by observing the necessary information (e.g., the channel condition, the transmission power, and specific QoS requirements) on the other types of players. Here, the preferences of CUs over DUs are defined as monetary payment from the DUs or the incurred interference on CUs. The DUs build their preferences over CUs based on the channel conditions or achievable transmission rate. A CU-DU matching is said to be unacceptable if the system's QoS requirements are violated. Players that are unacceptable are then removed from each other's preferences. Then each player (CU or DU) sorts the acceptable players in descending order of its preferences. After setting up the preferences, proper matching algorithms must be developed to achieve the required system objectives such as maximizing the throughput. For example, when using the DA algorithm, as explained in Fig. 2, DUs will propose to the CUs who, in turn, will accept or reject the received applications. The complexity of this iterative process depends on the total number of acceptable pairs $m$, which is $\mathcal{O}(m)$ [8].

However, beyond considering a global utility, in some scenarios, one is interested in optimizing the performance of one of the two sets of players, such as the DUs. Based on our work in [15], we proposed the idea of incorporating a form of "cheating" in the preferences in an effort to improve the DUs' utilities. Cheating is done by enabling the DUs to smartly change their preferences so as to reap more performance gains. As shown in our result in Fig. 6, the use of such cheating strategies can improve the DUs' utility while simultaneously improving the system utility, compared to a DA algorithm. In addition, as done in [6], one can extend such D2D models to cases in which D2D communication must explore, beyond physical layer parameters, the social ties of the users. Here, as shown in [6], one can cast the problem as a class II problem to capture peer effects that reflect how socially connected the users are who utilize D2D communication with an anchor device (i.e., used for content distribution or caching by the BSs). For such a model, one can enhance the DA algorithm to account for peer effects and show its convergence to a twosided stable matching.

D2D is undoubtedly an important application area for matching theory with a promising set of future problems.

\section{CONCLUSION}

inf increased computation complexity at the BS level. Formulating the D2D problem as a noncooperative game will be limited by the fact that it will still rely on individual stability and the need for D2D users to observe the preferences of other players. To counter these limitations, it is of interest to develop matching-theoretic models for D2D communications. To this end, we observe that D2D typically involves two types of users: cellular users (CUs) and D2D users (DUs). In the underlay mode of D2D operation, the CUs are exclusively assigned licensed spectrum chunks from the BSs, while the DUs must
In this article, we have provided the first comprehensive tutorial on using matching theory for developing innovative resource management mechanisms in wireless networks. First, we have provided the fundamental concepts of matching theory and discussed a variety of properties that allow the definition of several classes of matching scenarios. Then we have proposed three new that can be adopted in wireless networking environments. For each class, we have developed the basic concepts and solutions that can be used to engineering-oriented classes of matching theory 
address related problems. We have provided a detailed treatment on how to use such matchingtheoretic tools in specific wireless applications. In a nutshell, this article is expected to provide an accessible and holistic tutorial on the use of new techniques from matching theory for addressing pertinent problems in emerging wireless systems.

\section{REFERENCES}

[1] Z. Han et al., Game Theory in Wireless and Communication Networks: Theory, Models and Applications, Cambridge Univ. Press, Oct. 2011.

[2] E. A. Jorswieck, "Stable Matchings for Resource Allocation in Wireless Networks," Proc. 17th Int'l. Conf. Digital Signal Processing, Corfu, Greece, July 2011

[3] A. Leshem, E. Zehavi, and Y. Yaffe, "Multichannel Opportunistic Carrier Sensing for Stable Channel Access Control in Cognitive Radio Systems," IEEE JSAC, vol. 30, no. 1, Jan. 2012, pp. 82-95.

[4] O. Naparstek, A. Leshem, and E. A. Jorswieck, "Distributed Medium Access Control for Energy Efficient Transmission in Cognitive Radios," arXiv preprint arXiv:1401.1671, 2014

[5] F. Pantisano et al., "Matching with Externalities for Context-Aware User-Cell Association in Small Cell Networks," Atlanta, GA, Dec. 2013

[6] O. Semiari et al., "On Self-Organizing Resource Allocation for Social Context-Aware small cell networks," Proc. 1st KuVS Wksp. Anticipatory Networks, Stuttgart, Germany, Sept. 2014.

[7] A. Roth and M. A. O. Sotomayor, Two-Sided Matching: A Study in Game-Theoretic Modeling and Analysis, Cambridge Univ. Press, Mar. 1992.

[8] D. F. Manlove, Algorithmics of Matching Under Preferences, World Scientific, 2013.

[9] R. W. Irving, P. Leather, and D. Gusfield, "An Efficient Algorithm for the Optimal Stable Marriage," J. ACM vol. 34, no. 3, July 1987, pp. 532-43.

[10] H. Xu and B. Li, "Seen as Stable Marriages," Proc. IEEE INFOCOM, Shanghai, China, Mar. 2011.

[11] D. Gale and L. S. Shapley, "College Admissions and the Stability of Marriage," Amer. Math. Monthly, vol. 69 , no. 1, Jan. 1962 , pp. 9-15.

[12] K. Hamidouche, W. Saad, and M. Debbah, "Many-toMany Matching Games for Proactive Social-Caching in Wireless Small Cell Networks," Proc. 12th Int'l. Symp. Modeling and Optimization in Mobile, Ad Hoc, and Wireless Networks, Hammamet, Tunisia, May 2014.

[13] S. Bayat et al., "Multiple Operator and Multiple Femtocell Networks: Distributed Stable Matching," IEEE ICC Ottawa, Canada, June 2012.

[14] W. Saad et al., "A College Admissions Game for Uplink User Association in Wireless Small Cell Networks," Proc. IEEE INFOCOM, Toronto, Canada, Apr.-May 2014.

[15] Y. Gu et al. "Cheating in Matching of Device to Device Pairs in Cellular Networks," Proc. IEEE GLOBECOM, Austin, TX, Dec. 2014.

\section{BIOGRAPHIES}

YUNAN Gu (ygu6@uh.edu) received her B. S. degree in electronic engineering from Nanjing University of Science and Technology in June 2011 and her M. S. in computer science from Texas Southern University in 2013. Now, she is a Ph.D student in the Electronic and Computer Engineering Department at the University of Houston. Her research interests include wireless communications and matching theory.

WALID SAAD (walids@vt.edu) received his B.E., M.E., and Ph.D. degrees in computer and communications engineer ing from the Lebanese University in 2004, the American University of Beirut (AUB) in 2007, and the University of Oslo in 2010, respectively. Currently he is an assistant professor at the Bradley Department of Electrical and Computer Engineering at Virginia Tech. His research interests include wireless and social networks, game theory, cybersecurity, cyber-physical systems, and cognitive radio networks.

MEHDI BENNIs (bennis@ee.oulu.fi) received his M.Sc. degree from the Ecole Polytechnique Federale de Lausanne (EPFL), Switzerland, and the Eurecom Institute, France, in 2002. In 2004, he joined the Centre for Wireless Communications at the University of Oulu, Finland, as a research scientist. He obtained his Ph.D in December 2009 on spectrum sharing for future mobile cellular systems. His research interests include radio resource management, heterogeneous and small cell networks, game theory, and machine learning. $\mathrm{He}$ is currently the Principal Investigator of the European CELTIC project SHARING.

MÉrouane DebBah (merouane.debbah@huawei.com) entered the Ecole Normale Supérieure de Cachan, France in 1996, where he received his M.Sc and Ph.D. degrees. From 2003 to 2007, he was with the Mobile Communications department of the Institut Eurecom, Sophia Antipolis France, as an assistant professor. Since 2007, he has been a full professor at Supelec. Since 2014, he is vice-president of the Huawei France R\&D center and director of the Mathematical and Algorithmic Sciences Lab. His research interests are in information theory, signal processing, and wireless communications.

ZHU HAN [S'01, M'04,SM'09,F'14] (zhan2@uh.edu) received his B.S. degree in electronic engineering his Tsinghua University in 1997, and the M.S. and Ph.D. degrees in electrical engineering from the University of Maryland, College Park, in 1999 and 2003, respectively. Currently, he is an associate professor in the Electrical and Computer Engineering Department at the University of Houston, Texas. His research interests include wireless resource allocation and management, wireless communications and networking, game theory, wireless multimedia, security, and smart grid communication.
As D2D users may

share spectral bands

with one another as

well as with the cel-

lular network, the

introduction of D2D

in cellular networks

will lead to new chal-

lenges in terms of

interference manage-

ment and resource

allocation. Thus, it

will provide an

important application

for matching theory. 